\title{
Temperature and Pressure Behaviours of Methanol, Acetonitrile/Water Mixtures on Chromatographic Systems
}

\author{
Talal Aburjai, Muhammed Alzweiri*, Yusuf M. Al-Hiari \\ Faculty of Pharmacy, The University of Jordan, Amman, Jordan \\ E-mail: *m.alzweiri@ju.edu.jo \\ Received September 9, 2011; revised October 17, 2011; accepted October 26, 2011
}

\begin{abstract}
Temperature and pressure were shown to vary significantly with solvent mixing, showing maxima at different solvent ratios. Acetonitrile/water mixing resulted in temperature reduction of solutions whereas methanol/water mixing caused temperature increases. On the other hand, maximum recorded chromatographic pressure of acetonitrile:water mixtures occurred at a solvent ratio of 1:6 compared with methanol:water, which showed a maximum pressure at a solvent ratio of 1:1. These findings can be of use in stabilizing retention time shifts during HPLC-based studies associated with compound identification based on retention time such as analysis of complex mixtures.
\end{abstract}

Keywords: Retention Time, Pressure, Temperature, Methanol and Acetonitrile

\section{Introduction}

Methanol/water and acetonitrile/water mixtures are commonly used in chromatographic mobile phases. Many physical characteristics used to understand solvent behavior within these mixtures, for example excess molar volume and enthalpy change, have been reported intensively in the literature [1-3]. Careful inspection in the literature concerning solvent mixture interaction provokes a corner stone conclusion. Measurement of physical properties depicts nonlinear relation with the composition of mixtures. This might reflect the diversity of possible interactions of solvent molecules

Understanding temperature and pressure behavior influencing retention time shift in gradient systems of chromatography can be utilized to minimize the effect of retention time shift on molecular identification.

Retention time shift in chromatographic separations has undesirable effects on the results regardless of whether a target molecule or a profiling analysis approach was used. Minimizing the shift becomes more essential when the profiling analysis is being carried out accompanied by chemometrics, in order to avoid the result of biasing the data [4-6]. In spite of the availability of many software applications used to correct this shift, the solutions are not always satisfactory [7-11]. Thus, it is important to design HPLC mobile phases able to minimize retention time shift by controlling, temperature fluctuation and the excessive pressure of HPLC system. Particularly, during the application of gradient systems $[12,13]$.

\section{Experimental}

\subsection{Materials}

Acetonitrile and water were HPLC grade (VWR International Ltd., Lutterworth, UK). A Minitherm HI 8751 thermosensor (Hanna instruments, Romania) was used to measure the change of temperature of mixtures. Chromatographic pressure study was carried out by using Spectra system P2000 HPLC pump (Thermo Separation). HPLC columns used were ACE C8 $50 \times 3 \mathrm{~mm} 3 \mu \mathrm{m}$ and Kromasil 5ODS (C18) $250 \times 3.2 \mathrm{~mm} 5 \mu \mathrm{m}$.

\subsection{Procedures}

HPLC backpressure was measured for two reversed phases; $\mathrm{C} 8$ and $\mathrm{C} 18$, as the most common types of columns used in HPLC systems, against several ratios of methanol and acetonitrile in water with a constant flow rate of $0.7 \mathrm{~mL} / \mathrm{min}$. The pressure readings were taken directly from the LCD display of the HPLC pump.

The temperature change due to mobile phase solvent mixing was studied by titrating water with either methanol or acetonitrile and measuring the temperature at dif- 
ferent ratios of solvents. The procedure was repeated but by titrating the organic solvents with water instead in order to take in consideration the anomaly of molecular arrangement in mixtures at different solvent ratios.

\section{Results and Discussion}

One of the important chromatographic parameters that contribute to retention time variability is the column pressure, especially if a long analysis time is used. Thus pressure changes of examples of reversed phase columns were studied versus the organic modifier content in the mobile phase. As the solvent gradient affects pressure it was important to establish optimum conditions for analysis by assessing pressures under both gradient and isocratic conditions. For this reason a gradient run from zero to $100 \%$ was used in order to determine an approximate maximum value for column pressure. Following this, elution was carried out isocratically in order to accurately establish the solvent ratio that resulted in the maximum pressure value for different solvent/column conditions (Figures 1 and 2). While methanol induced the highest pressure in the reversed phase columns at a

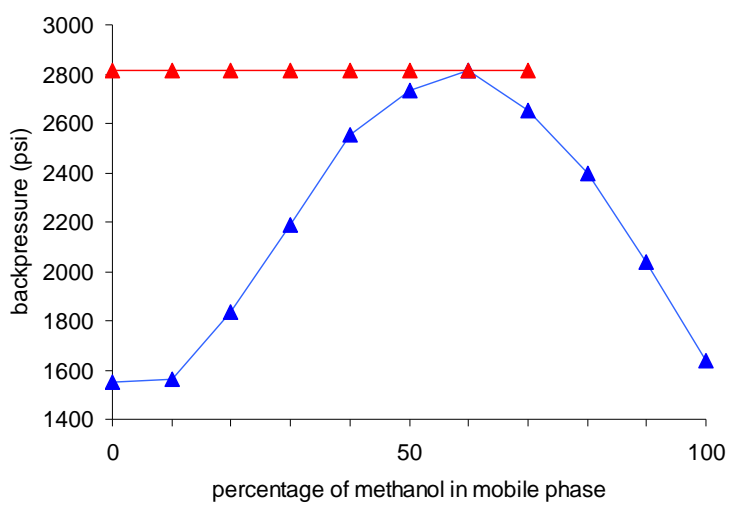

(a)

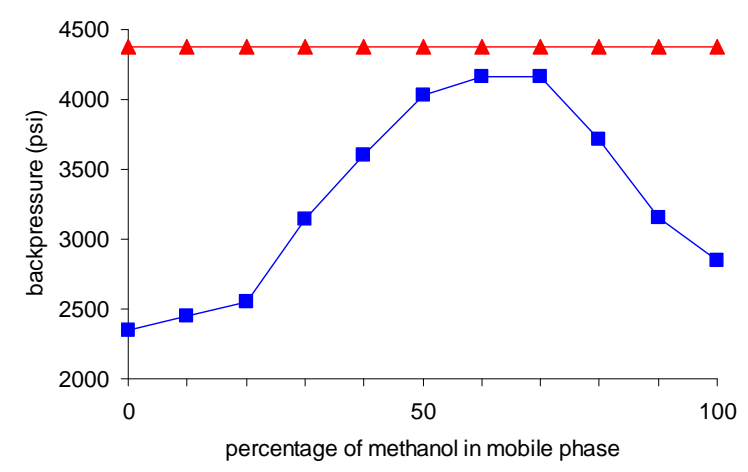

(b)

Figure 1. HPLC backpressure of reversed phase columns exposed to methanol-water mobile phases. (a) ACE C8 column; (b) Kromasil 5 ODS. (a) gradient of methanol and water, while $(\Delta)$ isocratic $(45: 55)$ methanol:water.

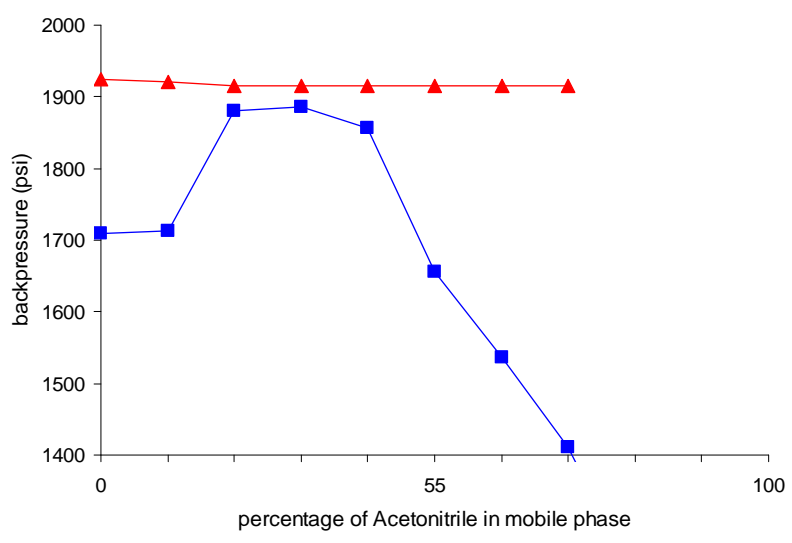

(a)

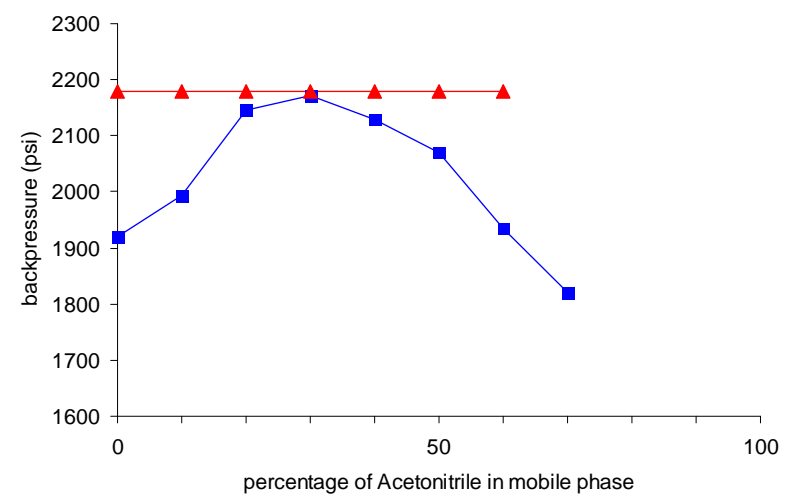

(b)

Figure 2. HPLC backpressure of reversed phase columns exposed to Acetonitrile-water mobile phases. (a) ACE C8 column; (b) Kromasil 5 ODS. (a) gradient of acetonitrile and water, while $(\Delta)$ isocratic $(\mathbf{1 5 : 8 5})$ acetonitrile:water.

methanol:water ratio of 45:55, acetonitrile produced its maximum back-pressure at an acetonitrile:water ratio of 15:85. However, this not only represents methanol-water or acetonitrile-water interactions but may also reflect the interaction of the mobile phase with the stationary phase of reversed phase columns. Methanol has the character of accepting and donating hydrogen bonds, while acetonitrile only accepts hydrogen bonds. Thus, stronger interactions between water and methanol molecules are expected as well as other interactions between methanol molecules and silica silanol groups are speculated. These interactions of methanol resist fluid flow through the HPLC column and consequently higher back pressures of methanol/water systems were obtained in comparison with acetonitrile/water systems. Comparison of Figure 1(a) with Figure1(b) depicts higher back pressure obtained from C18 column compared with C8 column. This might be due to more availability of silanol groups on the surface of $\mathrm{C} 18$ columns resulted from limited surface coverage during derivatization by large $\mathrm{C} 18$ molecules. Furthermore, Figure 2(b) shows higher back pressure 
comparing with Figure 2(a) due to the same reason mentioned previously. However, the difference of backpressure is with less extent in case of acetonitrile. This might be due to the limited interactions of acetonitrile with silanol groups in comparison with methanol. Moreover, distribution of data points of backpressure produced from methanol/water systems is closer to normal distribution, while acetonitrile/water has a significant deviation from the norm. This is due to inconsistency of water/acetonetrile interactions resulted from acetonitrile ability in forming clathrates with water as proved by our team [14].

Another factor affecting retention time is the temperature change due to mobile phase solvent mixing. Thus temperature change was studied by simple titration of methanol with water and vice versa, the same procedure being repeated for acetonitrile and water.

In the case of methanol/water mixing a considerable rise in temperature was detected up to approximately $40 \%$ contribution of either solvent to the mixtures (Figure 3(b)). This might indicate a relation between the formation of new hydrogen bonds among water and methanol molecules within the liquid with consequent decrease in entropy. Methanol/water mixtures did not show hysteresis in the thermal behavior during mixing procedure. Whether the addition started with water or with methanol, the system produced identical thermal behaviors. In contrast the mixing of acetonitrile/water caused a fall in temperature when a constant volume of acetonitrile titrated drop-wise with water (Figure 3(a)). This might indicate the increased disorder within the liquid and the overall breaking of hydrogen bonds. However, it is clear that there is a different behavior when water titrated with acetonitrile indicating several possibilities of arrangement between water and acetonitrile molecules depending on their relative molar ratios. These findings match NMR and IR studies of acetonitrile/water systems conducted by Alzweiri et al. [14]. It was also observed that drop in temperature occurred when water titrated with acetonitrile until acetonitrile reached $20 \%$ of the mixture then rapid increase in temperature was occurred. This might be due to possible clathrate formation between water and acetonitrile at certain molar ratios. Anomaly of thermal behavior resulted from Water titrated with acetonitrile compared with acetonitrile titrated with water matches Satoh and Nakanishi suggestion in existing different arrangements of water and acetonitrile in mixtures [15].

Variations in temperature and backpressure show that there are critical solvent ratios at which these physicochemical properties change dramatically. The knowledge of these critical ratios might be exploitable for controlling retention time shifts in different chromatographic systems.

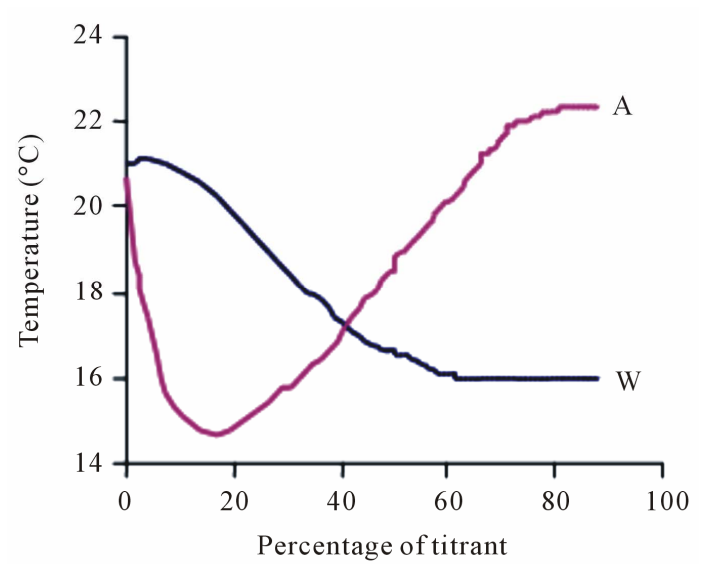

(a)

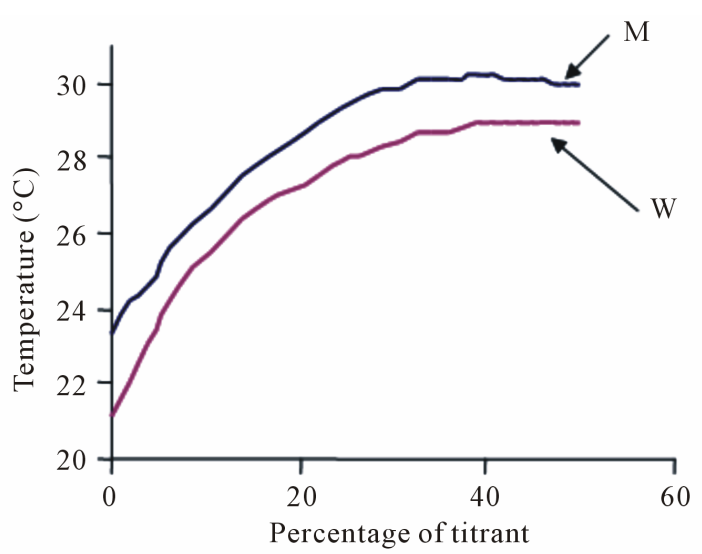

(b)

Figure 3. Temperature change of water titrated drop wise with acetonitrile ((a)-A), acetonitrile titrated with water ((a)-W), water titrated with methanol ((b)-M) and methanol titrated with water $((b)-W)$.

\section{Conclusions}

Retention time shift of chromatographic peaks is a particular source of errors in molecular identification. In this study an attempt has been made to examine the pressure and temperature factors that influence retention time shift to allow future development of LC methods that would reduce intrinsic shift errors to a minimum. Temperature and pressure variation revealed that critical maxima occur during solvent mixing which can be taken in consideration during the setup of the chromatographic development. Despite the close physicochemical properties of acetonitrile and methanol, differences were observed in their behaviour and response as follows: 1) while acetonitrile/water mixing resulted in temperature reduction of the solution, methanol/water mixing caused temperature increase; 2) the maximum recorded column backpressure for an acetonitrile:water mixture occurred when the solvent ratio was 1:6, whereas a methanol:water 
mixture resulted in a maximum backpressure at a solvent ratio of $1: 1$.

\section{References}

[1] E. Bulemela, P. Tremaine and S.-I. Ikawa, "Volumetric Behavior of Water-Methanol Mixtures in the Vicinity of the Critical Region," Fluid Phase Equilibria, Vol. 245, 2006, p. 125.

[2] K. F. Ita McStravick, J. Lambert, N. Teahan and W. Earle Waghorne, "Enthalpy of Transfer of $-\mathrm{CH}_{2}$ - between Water and Organic Solvents or Mixed Aqueous Organic Solvents or Mixed Aquoues Organic Solvent Systems," Journal of Molecular Liquids, Vol. 94, 2001, pp. 145153.

[3] G. R. Behbehani, S. Ghammamy and W. E. Waghorne, "Enthalpies of Transfer of Acetonitrile from Water to Aqueous Methanol, Ethanol and Dimethylsulphoxide Mixtures at $298.15 \mathrm{~K}$," Thermochimica Acta, Vol. 448, No. 1, 2006, pp. 37-40. doi:10.1016/j.tca.2006.06.021

[4] M. Vosough and A. Salemi, "Second-Order Standard Addition for Deconvolution and Quantification of Fatty Acids of Fish Oil Using GC-MS," Talanta, Vol. 73, No. 1, 2007, pp. 30-36. doi:10.1016/j.talanta.2007.02.025

[5] M. Bechtold, A. Felinger, M. Held and S. Panke, "Adsorption Behavior of a Teicoplanin Aglycone Bonded Stationary Phase under Harsh Overload Conditions," Journal of Chromatography A, Vol. 1154, No. 1-2, 2007, pp. 277-286. doi:10.1016/j.chroma.2007.03.103

[6] D. Bylund, R. Danielsson, G. Malmquist and K. E. Markides, "Chromatographic Alignment by Warping and Dynamic Programming as a Pre-Processing Tool for PARAFAC Modelling of Liquid Chromatography-Mass Spectrometry Data," Journal of Chromatography A, Vol. 961, No. 2, 2002, pp. 237-244. doi:10.1016/S0021-9673(02)00588-5

[7] K. M. Pierce, B. W. Wright and R. E. Synovec, "Unsupervised Parameter Optimization for Automated Retention Time Alignment of Severely Shifted Gas Chromatographic Data Using the Piecewise Alignment Algorithm," Journal of Chromatography A, Vol. 1141, No. 1, 2007, pp. 106-116. doi:10.1016/j.chroma.2006.11.101

[8] F. Gong, Y.-Z. Liang, Y.-S. Fung and F.-T. Chau, "Correction of Retention Time Shifts for Chromatographic Fingerprints of Herbal Medicines," Journal of Chromatography A, Vol. 1029, No. 1-2, 2004, pp. 173-183. doi:10.1016/j.chroma.2003.12.049

[9] F. O. Andersson, R. Kaiser and S. P. Jacobsson, "Data Preprocessing by Wavelets and Genetic Algorithms for Enhanced Multivariate Analysis of LC Peptide Mapping," Journal of Pharmaceutical and Biomedical Analysis, Vol. 34, No. 3, 2004, pp. 531-541. doi:10.1016/S0731-7085(03)00583-1

[10] K. J. Johnson, B. W. Wright, K. H. Jarman and R. E. Synovec, "High-Speed Peak Matching Algorithm for Retention Time Alignment of Gas Chromatographic Data for Chemometric Analysis," Journal of Chromatography A, Vol. 996, No. 1-2, 2003, pp. 141-155. doi:10.1016/S0021-9673(03)00616-2

[11] A. M. van Nederkassel, M. Daszykowski, P. H. C. Eilers and Y. V. Heyden, "A Comparison of Three Algorithms for Chromatograms Alignment," Journal of Chromatography A, Vol. 1118, No. 2, 2006, pp. 199-210. doi:10.1016/j.chroma.2006.03.114

[12] M. Makela and L. Pyy, "Effect of Temperature on Retention Time Reproducibility and on the Use of Programmable Fluorescence Detection of Fifteen Polycyclic Aromatic Hydrocarbons," Journal of Chromatography A, Vol. 699, No. 1-2, 1995, pp. 49-57. doi:10.1016/0021-9673(95)00120-C

[13] W. S. Gardner, H. A. Bootsma, C. Evans and P. A. S. John, "Improved Chromatographic Analysis of 15N:14N Ratios in Ammonium or Nitrate for Isotope Addition Experiments," Marine Chemistry, Vol. 48, No. 3-4, 1995, pp. 271-282. doi:10.1016/0304-4203(94)00060-Q

[14] M. Alzweiri, J. Parkinson, D. Watson and S. Steer, "Microscopic Trends in Methanol/Water and Acetonitrile/ Water Systems," Jordan Journal of Pharmaceutical Sciences, Vol. 4, 2011, pp. 20-28.

[15] Y. Satoh and K. Nakanishi, "Theoretical Studies of Acetonitrile-Water Mixtures/Monte Carlo Simulation," Fluid Phase Equilibria, Vol. 104, 1995, pp. 41-55. doi:10.1016/0378-3812(94)02638-H 